\title{
IN-PLANE OSCILLATIONS OF A RING DRIVEN BY A SOAP FILM CATENOID
}

\section{PAVEL GRINFELD}

\section{Communicated by Gregory Naber}

\begin{abstract}
In this paper, we calculate the frequency of small oscillations of a ring confined to a plane that, along with a fixed ring of the same size, supports a soap film catenoid. The restoring force is provided by the film's surface tension. We assume that the soap film is massless and continuously assumes the shape of minimal area. Mathematically, the problem is equivalent to calculating the second derivative of the total area with respect to the displacement of the ring. The calculus of moving surfaces is extensively used in the presented calculation.
\end{abstract}

\section{Introduction}

In this paper we propose to study the harmonic oscillations of a ring confined to a plane under the influence of a massless soap film that continuously assumes the surface of minimum area. The presented calculation demonstrates the stability of the equilibrium catenoid with respect to an in-plane shift of the supporting rings. The calculation relies on the calculus of moving surfaces.

The catenoid has historically played an important role in the calculus of variations and the study of minimal surfaces. In 1744, Euler showed that the catenoid is a minimal surface of revolution in his celebrated work on the calculus of variations [2]. Since then, a number of embedded (that is, non-self-intersecting) surfaces have been discovered analytically: helicoid by Jean Meusnier in 1776, Scherk surfaces by Heinrich Scherk in 1834, Riemann surfaces in 1860 and the Schwarz quadrilateral in 1890. In recent decades, a number of minimal surfaces of genus greater than zero have been discovered, including surfaces by Enneper, Catalan, Henneberg and Costa. Minimal surfaces continue to be an active area of research (see the works of Meeks, for example [6] and references therein).

Minimal surfaces play an important role in modern physical applications including the study of fluid films [1], polymer networks [10], crystallography and protein structures [5] and smectic-A [7] and other liquid crystal phases. 


\section{Description of the Problem}

Consider the physical system in Fig. 1 consisting of two coaxial rings of radius $R$ a distance $H$ apart. The top ring is fixed while the bottom ring slides on a frictionless substrate. It is kept on the substrate by the force of gravity or some other mechanism which is inconsequential for our discussion. If the bottom ring is displaced from the equilibrium, the soap film provides the restoring force. What is the frequency of the resulting small oscillations?

In this paper we solve a geometric problem that is directly related to this question. Namely, assuming that the bottom ring is shifted by $L$ and the area of the resulting minimal surface is $A(L)$ we calculate $A^{\prime \prime}(0)$. Note that the first derivative vanishes by symmetry.

\subsection{The Equilibrium Catenoid}

Point the $z$-axis (or, as we refer to it, the $Z^{3}$-axis) downward, place the top ring at $z=0$ and the bottom ring at $z=H$. The equilibrium catenoid that spans coaxial rings of equal radius $R$ (see Fig.1) is given in cylindrical coordinates by

$$
r(z)=a \cosh \frac{z-H / 2}{a}
$$

where the constant $a$ is selected to match the radius of the ring at each end

$$
a \cosh \frac{H}{2 a}=R \text {. }
$$

Let $\mu$ be the critical value above which the equation

$$
\cosh \mu x=x
$$

has no solutions. The approximate numerical value of $\mu$ is 0.66274 . For any value of $H<2 R \mu$, equation (2) has two solutions. The solution that corresponds to the smaller value of $a$ is morphologically unstable. The other one is stable and is the object of our study.

Let $\Phi$ be the angle that the equilibrium catenoid forms with the vertical axis, as illustrated in Fig.1. Then

$$
\cos \Phi=\frac{a}{R} \quad \text { and } \quad \sin \Phi=\tanh \frac{H}{2 a} .
$$

Therefore, for a given value of $R$, the parameters $H, a$, and $\Phi$ are uniquely related and we shall use them interchangeably. 

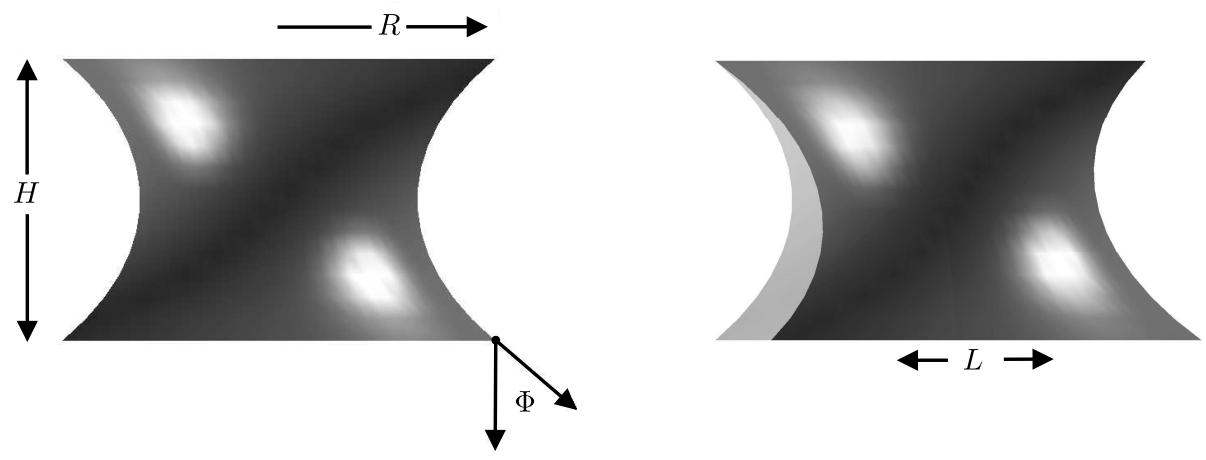

Figure 1. The equilibrium catenoid and a minimal surface spanning the original top ring and the bottom ring shifted by a distance.

Looking ahead, we will derive the following expression for the second derivative $A^{\prime \prime}(0)$ of $A$ at $L=0$

$$
A^{\prime \prime}(0)=\frac{\pi a^{2}}{R^{2}}\left(\frac{2+2 \cosh \frac{H}{a}-\frac{H}{a} \sinh \frac{H}{a}}{\left(1+\cosh \frac{H}{a}\right)\left(\frac{H}{a}+\sinh \frac{H}{a}\right)}+\tanh \frac{H}{2 a}\right) .
$$

The plot of $A^{\prime \prime}(0)$ as a function of $H$ for $R=1$ is seen in Fig.2. We observe first that $A^{\prime \prime}(0)$ is positive for all values of $H$ indicating stability with respect to the horizontal displacement of the bottom ring. Secondly, $A^{\prime \prime}(0)$ approaches infinity as $H \rightarrow 0$. In fact

$$
A^{\prime \prime}(0)=\frac{\pi}{H}+O\left(\frac{1}{H^{3}}\right) \text { as } H \rightarrow 0
$$

as one would expect, since the catenoid is a nearly flat cylinder for small $H$, for which equation (6) is easily verified.

\section{Calculation}

\subsection{Variation of Area}

The calculation is conducted in the framework of the calculus of moving surfaces (CMS). For the description of this technique, see [3] and references therein. The CMS has so far been developed for hypermanifolds embedded in Euclidean spaces. Our analysis involves two such surfaces. First, the catenoid $S$ is embedded in the three dimensional space and has a rigid moving contour boundary $s$, that is the bottom ring. Second, the motion of the rigid contour boundary $s$, will be analyzed 


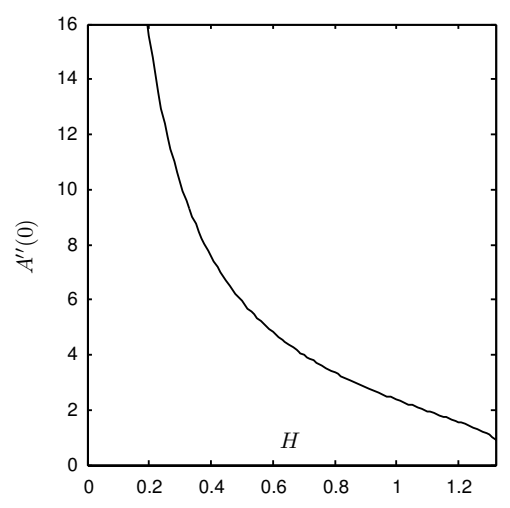

Figure 2. The plot of $A^{\prime \prime}(0)$ in equation (5) as a function of $H$ for $R=1$ and $0<H \leq 2 \mu R$, where $\mu$ is the largest value for which equation (3) has a solution. Consequently, the value $H=2 \mu R$ is the largest value of $H$ for which a catenoid solution exists.

from the point of view of the embedding within its plane. In the course of our calculation we will need to obtain the rate of change of the catenoid surface normal $\mathbf{N}$ with respect to the motion of the bottom ring. This will require a "crossover" formula that relates the $\delta / \delta t$-derivative in space with the $\delta_{P} / \delta t$-derivative in the plane. When applied to contour restrictions $F$ of surface invariants, such as $\mathbf{N}$, the two derivatives are related by the chain rule

$$
\frac{\delta_{P} F}{\delta t}=\frac{\delta F}{\delta t}+c n^{\alpha} \nabla_{\alpha} F
$$

where $n^{\alpha}$ is the contour normal that lies within the tangent plane to the surface $S$ and $c$ is the invariant velocity of the contour with respect to $S$. The velocity $c$ was discussed in [4].

The starting point of our calculation is the following law that governs the rate of change of the integral $\int_{S} T \mathrm{~d} S$ over a deforming surface $S$ with a moving contour boundary $s$

$$
\frac{\mathrm{d}}{\mathrm{d} t} \int_{S} T \mathrm{~d} S=\int_{S} \frac{\delta T}{\delta t} \mathrm{~d} S-\int_{S} T C B_{\alpha}^{\alpha} \mathrm{d} S+\int_{s} c T \mathrm{~d} s
$$

where $B_{\alpha}^{\alpha}$ (the trace of the curvature tensor $B_{\beta}^{\alpha}$ ) is the mean curvature and $C$ is the velocity of the surface $S$ with respect to the ambient Euclidean space.

The area $A$ of the catenoid is given by the integral

$$
A=\int_{S} \mathrm{~d} S
$$


We imagine a smooth evolution of the initial catenoid where the bottom ring moves to the right with uniformly velocity in time $t$ and the rest of the surface adjusts as to maintain the minimal property

$$
B_{\alpha}^{\alpha}=0 \quad \text { at all } t
$$

Then the first derivative of $A$ is obtained by an application of (8) to (9) with $T \equiv 1$. The result, valid it all times $t$, is

$$
\frac{\mathrm{d} A}{\mathrm{~d} t}=\int_{s} c \mathrm{~d} s .
$$

The second derivative is obtained by a repeated application of (8) to (11), but this time in the context of bottom ring $s$ moving within the plane

$$
\frac{\mathrm{d}^{2} A}{\mathrm{~d} t^{2}}=\int_{s} \frac{\delta_{P} c}{\delta t} \mathrm{~d} s-\int_{s} c C_{P} B_{\Psi}^{\Psi} \mathrm{d} s
$$

where $C_{P}$ (where $P$ is for plane) is the invariant velocity of the contour $s$ within the plane and $B_{\Psi}^{\Psi}$ is its mean curvature with respect to its embedding within the plane. By our convention, capital Greek letters have value one. With respect to the external normal $\mathbf{N}_{P}$, the mean curvature $B_{\Psi}^{\Psi}$ is given by [8], [9]

$$
B_{\Psi}^{\Psi}=-\frac{1}{R}
$$

\subsection{Differential Objects in the Plane}

We begin by calculating the contour velocity $C_{p}$ within the plane. It will later lead to the contour velocity $c$ with respect to the surface $S$ and to $\delta_{P} c / \delta t$. Refer the plane to Cartesian coordinates $P^{i}$. Refer the contour to the angle $S^{\Psi}=\theta$, where $\Psi=1$, as mentioned above. Then the evolution of the rigid contour, which is moving to the right with unit velocity, is

$$
P^{i}(t, \theta)=\left[\begin{array}{c}
t+R \cos \theta \\
R \sin \theta
\end{array}\right] .
$$

The shift tensor $Z_{\Psi}^{i}$, defined as $\partial P^{i} / \partial S^{\Psi}$, is

$$
Z_{\Psi}^{i}=\left[\begin{array}{r}
-R \sin \theta \\
R \cos \theta
\end{array}\right]
$$

Since the plane is referred to Cartesian coordinates, the shift tensor $Z_{i \Psi}$ with a covariant index $i$, has the same entries. 
The metric tensors $Z_{\Psi \Psi}=Z_{\Psi}^{i} Z_{i \psi}$ and $Z^{\Psi \Psi}$ (the inverse of $Z_{\Psi \Psi}$ ) are given by

$$
Z_{\Psi \Psi}=R^{2}, \quad Z^{\Psi \Psi}=\frac{1}{R^{2}}
$$

The velocity $v_{P}^{i}$, defined as $\partial P^{i} / \partial t$, and its projection $v_{P}^{\Psi}=Z^{\Psi \Lambda} Z_{i \Lambda} v_{P}^{i}$ onto $s$ are

$$
v_{P}^{i}=\left[\begin{array}{l}
1 \\
0
\end{array}\right] \quad \text { and } \quad v_{P}^{\Psi}=-\frac{1}{R} \sin \theta
$$

The components $N_{P}^{i}$ of the exterior normal $\mathbf{N}_{P}$ within the plane are easily guessed

$$
N_{P}^{i}=\left[\begin{array}{c}
\cos \theta \\
\sin \theta
\end{array}\right] .
$$

The covariant components $\left(N_{P}\right)_{i}$ have the same values.

The velocity $C_{P}$ is defined as the normal projection of $v_{P}^{i}$ onto $s$

$$
C_{P}=v_{P}^{i}\left(N_{P}\right)_{i}
$$

It can be described by the explicit expression

$$
C_{P}=\cos \theta \text {. }
$$

We have thus obtained one of the ingredients of equation (12). The derivative $\delta_{P} C_{P} / \delta t$ of $C_{P}$ is calculated directly by the definition of the $\delta_{P} / \delta t$-derivative

$$
\frac{\delta_{P} C_{P}}{\delta t}=\frac{\partial C_{P}}{\partial t}-v_{P}^{\Psi} \nabla_{\Psi} C_{P}=-\frac{1}{R} \sin ^{2} \theta .
$$

\subsection{Calculation of the Contour Velocity}

For a moment, we have to step out of the plane and consider the motion of the rigid contour boundary $s$ as embedded in the three dimensional Euclidean space. This motion is characterized by the vector velocity $\Gamma$ given by

$$
\boldsymbol{\Gamma}=C_{P} \mathbf{N}_{P}
$$

Then the contour velocity $c$ with respect to the surface $S$ is the tangential projection of $\boldsymbol{\Gamma}$ onto $S$

$$
c=C_{P} \mathbf{N}_{P} \cdot \mathbf{n} \text {. }
$$

A vector manipulation of the right hand side leads to a significant simplification. Suppose that $\mathbf{t}$ is the unit tangent vector to the contour $s$ within the plane. Then

$$
\mathbf{n}=\mathbf{t} \times \mathbf{N}
$$


and

$$
c=C_{P} \mathbf{N}_{P} \cdot(\mathbf{t} \times \mathbf{N}) .
$$

By an even permutation of the triple vector product, we have

$$
c=C_{P} \mathbf{N} \cdot\left(\mathbf{N}_{P} \times \mathbf{t}\right) .
$$

Both $\mathbf{N}_{P}$ and $\mathbf{t}$ lie in the plane of the ring. Therefore $\mathbf{N}_{P} \times \mathbf{t}$ is a unit vector pointing upwards, which, in our notation, is $-\mathbf{k}$. Thus

$$
c=-C_{P} \mathbf{N} \cdot \mathbf{k}
$$

We have thus obtained another ingredient from equation (12).

\subsection{Calculation of $\delta_{P} c / \delta t$}

Our starting point for the calculation of $\delta_{P} c / \delta t$ is equation (26). Apply the product rule

$$
\frac{\delta_{P} c}{\delta t}=-\frac{\delta_{P} C_{P}}{\delta t} \mathbf{N} \cdot \mathbf{k}-C_{P} \frac{\delta_{P} \mathbf{N}}{\delta t} \cdot \mathbf{k}
$$

Next, apply the chain rule (7) to the second term

$$
\frac{\delta_{P} c}{\delta t}=-\frac{\delta_{P} C_{P}}{\delta t} \mathbf{N} \cdot \mathbf{k}-C_{P}\left(\frac{\delta \mathbf{N}}{\delta t}+c n^{\beta} \nabla_{\beta} \mathbf{N}\right) \cdot \mathbf{k}
$$

and apply one rule from the differentiation table of the CMS

$$
\frac{\delta \mathbf{N}}{\delta t}=-\mathbf{S}^{\alpha} \nabla_{\alpha} C
$$

and one rule from classical tensor calculus [8], [9]

$$
\nabla_{\beta} \mathbf{N}=-B_{\alpha \beta} \mathbf{S}^{\alpha}
$$

where $\mathbf{S}^{\alpha}$ is the contravariant basis. So far, we have arrived at the following expression for $\delta_{P} c / \delta t$

$$
\frac{\delta_{P} c}{\delta t}=-\frac{\delta_{P} C_{P}}{\delta t} \mathbf{N} \cdot \mathbf{k}+C_{P}\left(\nabla_{\alpha} C+c n^{\beta} B_{\alpha \beta}\right) \mathbf{S}^{\alpha} \cdot \mathbf{k} .
$$

We must therefore now turn to the calculation of these differential objects on the catenoid: $C, \mathbf{S}^{\alpha}, B_{\alpha \beta}$, and $n^{\beta}$. 


\subsection{Differential Objects on the Catenoid}

Introduce Cartesian coordinates $Z^{i}$ in the ambient three dimensional Euclidean space. Let $Z^{1}$ and $Z^{2}$ correspond to the usual arrangement of Cartesian coordinates $x$ and $y$ in the plane, and let $Z^{3}$ point down. Parametrize the equilibrium catenoid with the help of two variables $S^{\alpha}, S^{1}=\gamma$ and $S^{2}=\theta$ as follows

$$
\begin{aligned}
& Z^{1}(\gamma, \theta)=a \cosh \frac{\gamma-H / 2}{a} \cos \theta \\
& Z^{2}(\gamma, \theta)=a \cosh \frac{\gamma-H / 2}{a} \sin \theta \\
& Z^{3}(\gamma, \theta)=\gamma
\end{aligned}
$$

where $a$ is determined by equation (2).

The shift tensor $Z_{\alpha}^{i}=\partial Z^{i} / \partial S^{\alpha}$ is

$$
Z_{\alpha}^{i}=\left[\begin{array}{cc}
\sinh \frac{\gamma-H / 2}{a} \cos \theta & -a \cosh \frac{\gamma-H / 2}{a} \sin \theta \\
\sinh \frac{\gamma-H / 2}{a} \sin \theta & a \cosh \frac{\gamma-H / 2}{a} \cos \theta \\
1 & 0
\end{array}\right]
$$

and the shift tensor $Z_{i \alpha}$ with a covariant index $i$ has the same entries.

The covariant metric tensor $S_{\alpha \beta}=Z_{\alpha}^{i} Z_{i \alpha}$ is

$$
S_{\alpha \beta}=\left[\begin{array}{cc}
\cosh ^{2} \frac{\gamma-H / 2}{a} & 0 \\
0 & a^{2} \cosh ^{2} \frac{\gamma-H / 2}{a}
\end{array}\right] .
$$

The contravariant metric tensor $S^{\alpha \beta}$, the matrix inverse of $S_{\alpha \beta}$, is

$$
S^{\alpha \beta}=\left[\begin{array}{cc}
\frac{1}{\cosh ^{2} \frac{\gamma-H / 2}{a}} & 0 \\
0 & \frac{1}{a^{2}} \frac{1}{\cosh ^{2} \frac{\gamma-H / 2}{a}}
\end{array}\right] .
$$

The normal $N^{i}$ is given by

$$
N^{i}=\frac{1}{\cosh \frac{\gamma-H / 2}{a}}\left[\begin{array}{c}
\cos \theta \\
\sin \theta \\
\sinh \frac{\gamma-H / 2}{a}
\end{array}\right] .
$$

The covariant curvature tensor $B_{\alpha \beta}$ is

$$
B_{\alpha \beta}=\left[\begin{array}{ll}
-\frac{1}{a} & \\
& a
\end{array}\right] .
$$


Raise the index $\alpha$ to obtain $B_{\beta}^{\alpha}$

$$
B_{\beta}^{\alpha}=\left[\begin{array}{cc}
-\frac{1}{a \cosh ^{2} \frac{\gamma-H / 2}{a}} & \\
& \frac{1}{a \cosh ^{2} \frac{\gamma-H / 2}{a}}
\end{array}\right] .
$$

The trace $B_{\alpha}^{\alpha}$ of $B_{\beta}^{\alpha}$, which gives the mean curvature is zero

$$
B_{\alpha}^{\alpha}=0
$$

This condition is satisfied not only by the equilibrium catenoid, but continuously throughout the evolution as expressed by equations (10). The Gaussian curvature $K$ is the determinant of $B_{\beta}^{\alpha}$

$$
K=-\frac{1}{a^{2} \cosh ^{4} \frac{\gamma-H / 2}{a}} .
$$

The trace $B_{\beta}^{\alpha} B_{\alpha}^{\beta}$ of the third fundamental form $B_{\beta}^{\alpha} B_{\gamma}^{\beta}$ is

$$
B_{\beta}^{\alpha} B_{\alpha}^{\beta}=-2 K
$$

and this relationship holds for any minimal surface. The Laplacian $\nabla_{\alpha} \nabla^{\alpha}$, best obtained by the Voss-Weyl equation, is

$$
\nabla_{\alpha} \nabla^{\alpha}=\frac{1}{\cosh ^{2} \frac{\gamma-H / 2}{a}}\left(\frac{\partial^{2}}{\partial \gamma^{2}}+\frac{\partial^{2}}{\partial \theta^{2}}\right)
$$

Next, we summarize the vector valued fields. With respect to the Cartesian basis $\mathbf{i}, \mathbf{j}, \mathbf{k}$, the position vector $\mathbf{R}$ is given by

$$
\mathbf{R}(\gamma, \theta)=a \cosh \frac{\gamma-H / 2}{a} \cos \theta \mathbf{i}+a \cosh \frac{\gamma-H / 2}{a} \sin \theta \mathbf{j}+\gamma \mathbf{k} .
$$

The covariant basis vectors $\mathbf{S}_{1}=\partial \mathbf{R} / \partial S^{1}$ and $\mathbf{S}_{2}=\partial \mathbf{R} / \partial S^{2}$ are given by

$$
\begin{aligned}
& \mathbf{S}_{1}=\sinh \frac{\gamma-H / 2}{a} \cos \theta \mathbf{i}+\sinh \frac{\gamma-H / 2}{a} \sin \theta \mathbf{j}+\mathbf{k} \\
& \mathbf{S}_{2}=-a \cosh \frac{\gamma-H / 2}{a} \sin \theta \mathbf{i}+a \cosh \frac{\gamma-H / 2}{a} \cos \theta \mathbf{j} .
\end{aligned}
$$

We next derive $\mathbf{n}$, the external unit normal to the bottom ring that lies within the catenoid's tangent plane. It is geometrically clear that $\mathbf{n}$ points along $\mathbf{S}_{1}$. Since $\mathbf{n}$ is unit length and the length of $\mathbf{S}_{1}$ at $\gamma=H$ is $\cosh (H / 2 a)$ or $R / a$, the components $n^{\alpha}$ of $\mathbf{n}$ are

$$
n^{\alpha}=\left[\begin{array}{c}
\frac{a}{R} \\
0
\end{array}\right]
$$


Finally, note that the combination $n^{\alpha} B_{\alpha \beta}$ is given by

$$
n^{\alpha} B_{\alpha \beta}=\left[\begin{array}{r}
-\frac{1}{R} \\
0
\end{array}\right] \text {. }
$$

Note that at the contour boundary

$$
\mathbf{N} \cdot \mathbf{k}=-\sin \Phi=-\tanh \frac{H}{2 a} .
$$

Therefore the contour velocity $c$ with respect to $S$, given in equation (26) is

$$
c=-C_{P} \mathbf{N} \cdot \mathbf{k}=\cos \theta \tanh \frac{H}{2 a} .
$$

Furtherer, $\mathbf{S}^{\alpha} \cdot \mathbf{k}$ vanishes for $\alpha=2$, while for $\alpha=1$, we have from (43)

$$
\mathbf{S}^{1} \cdot \mathbf{k}=\frac{1}{\cosh ^{2} \frac{H}{2 a}}=\frac{a^{2}}{R^{2}}
$$

The remaining undetermined element in equation (31) is the surface velocity $C$, which we will now calculate.

\subsection{Calculation of $C$}

The interior condition on $C$ is obtained by applying the $\delta / \delta t$-derivative to equation (10), which results in the differential equation

$$
\nabla_{\alpha} \nabla^{\alpha} C+C B_{\beta}^{\alpha} B_{\alpha}^{\beta}=0
$$

The boundary condition is determined by the motion of the bottom ring where $C$ must match the normal projection of $\boldsymbol{\Gamma}$

$$
C(H, \theta)=\boldsymbol{\Gamma} \cdot \mathbf{N}=\cos \theta \cos \Phi=\frac{a}{R} \cos \theta .
$$

At the top ring $\gamma=0$, the velocity $C$ must vanish

$$
C(0, \theta)=0
$$

In coordinate form, the differential equation (49) can be expressed with the help of (39) and (41)

$$
\frac{\partial^{2} C}{\partial \gamma^{2}}+\frac{1}{a^{2}} \frac{\partial^{2} C}{\partial \theta^{2}}+\frac{2}{a^{2} \cosh ^{2} \frac{\gamma-H / 2}{a}} C=0 .
$$


This equation can be solved by separation of variables. Let

$$
C(\gamma, \theta)=F(\gamma) \mathrm{e}^{\mathrm{i} m \theta}
$$

Then $F(\gamma)$ must satisfy the ordinary differential equation

$$
F^{\prime \prime}+\left(\frac{2}{a^{2} \cosh ^{2} \frac{\gamma-H / 2}{a}}-\frac{m^{2}}{a^{2}}\right) F=0
$$

whose general solution for $m=1$ (since we must match $\cos \theta$ ) is

$$
F(\gamma)=A \frac{1}{\sqrt{\cosh \frac{2 \gamma-H}{a}+1}}+B \frac{\sinh \frac{2 \gamma-H}{a}+\frac{2 \gamma-H}{a}}{\sqrt{\cosh \frac{2 \gamma-H}{a}+1}} .
$$

With all boundary conditions satisfied, $C(\gamma, \theta)$ is given by

$$
C(\gamma, \theta)=\frac{a}{R} \frac{\sqrt{1+\cosh \frac{H}{a}}\left(\sinh \frac{H}{a}+\frac{2 \gamma}{a}+\sinh \frac{2 \gamma-H}{a}\right)}{2 \sqrt{\cosh \frac{2 \gamma-H}{a}+1}\left(\frac{H}{a}+\sinh \frac{H}{a}\right)} \cos \theta .
$$

Its partial derivative $C_{\gamma}(\gamma, \theta)$ with respect to $\gamma$ at $\gamma=H$ is

$$
C_{\gamma}(H, \theta)=\frac{1}{R} \frac{2+2 \cosh \frac{H}{a}-\frac{H}{a} \sinh \frac{H}{a}}{\left(1+\cosh \frac{H}{a}\right)\left(\frac{H}{a}+\sinh \frac{H}{a}\right)} \cos \theta .
$$

\subsection{Putting Everything Together}

We now have in place all the elements of equation (31), repeated here

$$
\frac{\delta_{P} c}{\delta t}=-\frac{\delta_{P} C_{P}}{\delta t} \mathbf{N} \cdot \mathbf{k}+C_{P}\left(\nabla_{\alpha} C+c n^{\beta} B_{\alpha \beta}\right) \mathbf{S}^{\alpha} \cdot \mathbf{k} .
$$

The expression for $\delta_{P} C_{P} / \delta t$ is given in (21), that for $\mathbf{N} \cdot \mathbf{k}$ in (46) and that one for $C_{p}$ in (20). Since $\mathbf{S}^{\alpha} \cdot \mathbf{k}$ vanishes for $\alpha=2$, only the $\alpha=1$ component of the parenthesized expression is relevant. The quantity $\nabla_{\alpha} C=C_{\gamma}$ is given in (57), $c$ in (47), $n^{\beta} B_{\alpha \beta}$ in (45) and $\mathbf{S}^{1} \cdot \mathbf{k}$ in (48). Putting everything together we find

$$
\begin{aligned}
\frac{\delta_{P} c}{\delta t}= & \frac{1}{R} \sin ^{2} \theta \tanh \frac{H}{2 a} \\
& +\frac{1}{R} \cos ^{2} \theta\left(\frac{2+2 \cosh \frac{H}{a}-\frac{H}{a} \sinh \frac{H}{a}}{\left(1+\cosh \frac{H}{a}\right)\left(\frac{H}{a}+\sinh \frac{H}{a}\right)}+\tanh \frac{H}{2 a}\right) \frac{a^{2}}{R^{2}} .
\end{aligned}
$$


Integrating over the contour, we have

$$
\begin{aligned}
\int_{s} \frac{\delta_{P} c}{\delta t} \mathrm{~d} s= & \pi \tanh \frac{H}{2 a} \\
& +\pi\left(\frac{2+2 \cosh \frac{H}{a}-\frac{H}{a} \sinh \frac{H}{a}}{\left(1+\cosh \frac{H}{a}\right)\left(\frac{H}{a}+\sinh \frac{H}{a}\right)}+\tanh \frac{H}{2 a}\right) \frac{a^{2}}{R^{2}} .
\end{aligned}
$$

The remaining integral in the expression (12) for $A^{\prime \prime}(0)$ is

$$
-\int_{s} c C_{P} B_{\Psi}^{\Psi} \mathrm{d} s=-\pi \tanh \frac{H}{2 a} .
$$

Combining the two integrals, we obtain the final expression given in equation (5)

$$
A^{\prime \prime}(0)=\frac{\pi a^{2}}{R^{2}}\left(\frac{2+2 \cosh \frac{H}{a}-\frac{H}{a} \sinh \frac{H}{a}}{\left(1+\cosh \frac{H}{a}\right)\left(\frac{H}{a}+\sinh \frac{H}{a}\right)}+\tanh \frac{H}{2 a}\right)
$$

presented at the beginning of this paper.

\section{Discussion}

Equation (5) represents the second variation of area $A$ with respect to the horizontal displacement of the bottom ring, assuming that the surface $S$ continuously satisfies the minimal property (10). Its right hand side (5) is positive for all $H$ as evidenced by the plot in Fig. 2. Therefore the catenoid soap film is stable with respect to the in-plane dislocation of the supporting rings. This result can be used as a potential test for Laplace's model of capillary effects which essentially states that equilibrium and stability of soap film configurations is governed by the potential energy directly proportional to the total area. In a physical experiment, the expected frequency $f$ of small horizontal oscillations of the bottom ring of mass $M$ on a frictionless substrate is

$$
f=\sqrt{\frac{\sigma A^{\prime \prime}(0)}{M}}
$$

assuming that the total inertia of the soap film is negligible. It must also be true that the oscillations are small enough that the nonlinear dynamic features of fluid films, such as dynamic thickening, do not substantially interfere with the base mode.

The correctness of equation (5) can be tested numerically in a very effective away. It was discovered by Riemann in 1860 that the equilibrium surface that spans noncoaxial rings is composed of circular cross-sections. Let $r(z)$ denote the radius 
of the circle as a function of the $z$ coordinate and $s(z)$ denote the horizontal shift of the center of the circle. Then $r(z)$ and $s(z)$ are determined by the system of ordinary differential equations

$$
\begin{array}{r}
r r^{\prime \prime}-\left(r^{\prime}\right)^{2}-\left(s^{\prime}\right)^{2}-1=0 \\
r s^{\prime \prime}-2 r^{\prime} s^{\prime}=0
\end{array}
$$

subject to the boundary conditions

$$
\begin{aligned}
& r(0)=r(H)=R \\
& s(0)=0, \quad s(H)=L .
\end{aligned}
$$

This system can be solved in closed form. From the first equation it follows that

$$
s^{\prime}=a r^{2}
$$

where $a$ is a constant of integration, given by the equation

$$
L=a \int_{0}^{H} r^{2} \mathrm{~d} z .
$$

Therefore the system of ordinary differential equations (63) reduces to the single equation

$$
r r^{\prime \prime}-\left(r^{\prime}\right)^{2}-a r^{4}-1=0 .
$$

One integration (Greg Naber, private communication) yields

$$
\left(r^{\prime}\right)^{2}=a r^{4}+b r^{2}-1
$$

where $b$ is another constant of integration. A second integration yields

$$
r(z)=\frac{\mathrm{i} c \operatorname{sn}\left(\frac{\sqrt{2} a z}{c}-F\left(\mathrm{i} \operatorname{arcsinh} \frac{\sqrt{2} a}{c},-\frac{c^{4}}{4 a^{2}}\right),-\frac{c^{4}}{4 a^{2}}\right)}{\sqrt{2} a}
$$

where $c=\sqrt{b+\sqrt{4 a^{2}+b^{2}}}, \operatorname{sn}(u, k)$ is Jacobi's elliptic function, $F(u, k)$ is the elliptic integral of the first kind and the constants of integration are determined from the boundary conditions (64). The author has tested the solution (68) for a number of configurations.

On the other hand, equation (68) is quite complex and it is straightforward to solve (63) numerically by a shooting method. With the help of a numerical solution, the area of the skewed catenoid can be found to essentially arbitrary precision leading 
to an arbitrarily precise estimate for the second derivative $A^{\prime \prime}(0)$ which can then be compared with (5).

In conclusion, we would like to note the important question of morphological stability of the minimal surface that results for a finite displacement of the bottom ring. Such stability analysis entails the calculation of the second variation with respect to general morphological deformations of the surface and the subsequent determination of whether the second variation as positive for all such deformations.

\section{References}

[1] Boudaoud A. and Patricio P. and Ben Amar M., The Helicoid versus the Catenoid: Geometrically Induced Bifurcations, Phys. Rev. Lett. 83 (1999) 3836-3839.

[2] Euler L., Methodus inveniendi lineas curvas maximi minimive proprietate gaudentes, sive solutio problematis isoperimetrici lattissimo sensu accepti, 1744.

[3] Grinfeld P., Exact Nonlinear Equations for Fluid Films And Proper Adaptations of Conservation Theorems from Classical Hydrodynamics, J. Geom. Symm. Phys. 16 (2009) 1-21.

[4] Grinfeld P., Generalization of Hadamard's Laplace Eigenvalue Formula to Moving Embedded Manifolds, J. Geom. Symm. Phys. 15 (2009) 43-52.

[5] Hyde S., Andersson S., Larsson K., Blum Z., Landh T., Lidin S. and Ninham B., The Language of Shape, Elsevier, Amsterdam, 1997.

[6] Meeks W., The Structure of Stable Minimal Surfaces Near a Singularity, Michigan Math. J. 55 (2007) 155-161.

[7] Kamien R. and Lubensky T., Minimal Surfaces, Screw Dislocations, and Twist Grain Boundaries, Phys. Rev. Lett. 82 (1999) 2892-2895.

[8] Levi-Civita T., The Absolute Differential Calculus (Calculus of Tensors), Dover, New York, 1977.

[9] McConnell A., Applications of Tensor Analysis, Dover, New York, 1957.

[10] Rey A., Stability Analysis of Catenoidal Shaped Liquid Crystalline Polymer Networks, Macromolecules 30 (1997) 7582-7587.

Pavel Grinfeld

Department of Mathematics

271 Korman

Drexel University

Philadelphia, PA 19105

U.S.A.

E-mail address: pg@freeboundaries.com 